\title{
Adaptabilidad en la producción aeroespacial utilizando Sistemas Ciberfisicos
}

\begin{abstract}
RESUMEN
Una característica en la industria automatizada son los cambios de ingeniería y problemas de producción respecto a los mantenimientos correctivos y preventivos de los robots. Esto aunado a los problemas de faltantes de material y retrasos en el arribo de componentes; repercutiendo en pérdidas de tiempo de producción, que da como resultado planes de producción muy cambiantes, perdida de dinero y un estresante modo de operación en todos los departamentos. Este artículo describe cómo los sistemas ciberfisicos, son una herramienta importante paraunaindustria con ritmo acelerado; nuestro caso de estudio es la industria aeroespacial debido a su gran crecimiento en México. Actualmente a pesar de que se encuentran automatizadas las plantas aeroespaciales, el software que se utiliza en el proceso es independiente uno de otro, los componentes de software necesitan grados de libertad en forma de parámetros, para que puedan reaccionar a los nuevos requisitos que la industria aeroespacial requiere sin poner en riesgo la calidad.
\end{abstract}

Palabras-claves: Sistemas Ciber Físicos; Grados de libertad en software; Industria Aeroespacial.

ADAPTABILITY IN AEROSPACE PRODUCTION USING CyBERPHYSICAL SYSTEMS

\section{ABSTRACT}

A characteristic in the automated industry are the engineering changes and production problems with respect to the corrective and preventive maintenance of the robots. This coupled with the problems of missing material and delays in the arrival of components; resulting in lost production time, which results in very changing production plans, loss of money and a stressful mode of operation in all departments. This article describes how cyberphysical systems are an important tool for an industry with an accelerated rhythm; Our case study is the aerospace industry due to its great growth in Mexico. Currently, although aerospace plants are automated, the software used in the process is independent of each other the software components need degrees of freedom in the form of parameters, so that they can react to the new requirements that the industry aerospace requires without compromising quality.

Keywords: Cyber Physical Systems; Degrees of freedom in software; Aerospace Industry.

\section{INTRODUCCIÓN}

La problemática en la producción aeroespacial y en la mayoría de la industria electrónica radica en la capacidad de adaptación, es decir, una respuesta rápida y automática a los nuevos objetivos de producción. Cada vez que la producción se modifica, los requisitos de producción en toda la planta industrial repercutirán también. Se tomó como caso de estudio el área aeroespacial por el campo estricto de calidad y por el grado de robotización que utiliza. Según la federación mexicana de la industria aeroespacial (Federación Mexicana, 2016) en México se encuentran 270 empresas distribuidas en 18 estados de la República Mexicana (INEGI, 2016). Estas industrias cuentan con maquinaria de auto inserción, soldadura, corte etc. El área aeroespacial opera servicios de diseño e ingeniería, así como mantenimiento, reparación y operación. Los productos y servicios que se ofrecen en el país incluyen componentes del sistema de propulsión, aeroestructuras, componentes de los sistemas de aterrizaje, sistemas de frenado, partes mecanizadas de precisión, piezas de moldeo por inyección de plástico, tratamiento de superficies, partes eléctricas y electrónicas, piezas de material compuesto, diseño y servicios de ingeniería e interiores de aviones, entre otras. El desarrollo de nuevas tecnologías como el Internet de las cosas (Greengard, 2015) donde los objetos funcionan como sensores y realizan acciones activas con operación remota en tiempo real. También la tecnología de telefonía celular inteligente, equipados con GPS (Schwartz, 2015) han ayudado a el nuevo paradigma de los sistemas ciberfisicos; para el monitoreo y rastreo de componentes con proveedores en tiempo real. Además, actualmente los Smartphone se han convertido en sensores del entorno de manufactura.

Este artículo propone controlar mediante un sistema ciberfisico (Sang et al, 2015) el software utilizado en todos los departamentos del proceso industrial, esto a su vez controlara los robots del

Maestría en Ciencias de la Ingeniería. Docente del departamento de Sistemas y Comp. Tecnológico Nacional de México /ITMexicali. Email: veronicaquintero@itmexicali.edu.mx

2 Maestría en Electrónica. Docente del departamento Eléctrica- Electrónica. Tecnológico Nacional de México /ITMexicali. Email: mariocamarillo@itmexicali.edu.mx

3 Maestría en Computación. Docente del departamento de Sistemas y Comp. Tecnológico Nacional de México/ITMexicali. Email: fibañez@itmexicali.edu.mx

4 Maestría en Ingeniería Electrónica. Docente del departamento de Electrónica. Tecnológico Nacional de México /ITMexicali. Email: ggil@itmexicali.edu.mx

5 Maestría en Redes y Telecomunicaciones. Docente del departamento de Sistemas y Comp. Tecnológico Nacional de México/ITMexicali. Email: shernandez@itmexicali.edu.mx 6 Estudiante de Ingeniería en Sistemas Computacionales

Tecnológico Nacional de México/ITMexicali. Email: 01498754@itmexicali.edu.mx 
área de inserción automática utilizando metodologías y algoritmos modulares para modificar el plan de producción en tiempo real. En los últimos años, muchas soluciones de optimización para la producción han sido ampliamente estudiadas. El proceso de desarrollo basado en V-Modell XT (Kuhrmann et al,2016) que es una metodología de desarrollo de software; es una versión no comparable con CMMI (Beth, 2011) (modelo de procesos que contiene las mejores prácticas de la industria para el desarrollo, mantenimiento, adquisición y operación de productos y servicios). Mientras que CMMI solo describe qué se ha hecho, el Método-V describe el cómo, cuándo y quién es el responsable de haberlo hecho. El Método-V fue desarrollado para regular el proceso de desarrollo de software por la Administración Federal Alemana. Describe las actividades y los resultados que se producen durante el desarrollo del software. Las fases que agrupan estos procesos son las siguientes:
a. Análisis
b. Diseño
c. Programación
d. Prueba
e. Documentación
f. Mantenimiento
g. Reingeniería

\section{TRABAJO RELACIONADO}

Existe un proyecto de la unión europea llamado CONSENS (Kowther, 2016) el cual tiene como objetivo avanzar en las demandas de alta calidad en plantas continuas flexibles y dinámicas. Utiliza equipos de detección en línea de última generación y el control en lazo cerrado de los principales parámetros del producto. Se basa en la detección, control y monitoreo del desempeño transferible a procesos a gran escala, aplicando básicamente control y sensado. Como caso de estudio tiene una aplicación ciberfisica, dado que integran tres capas esenciales las cuales son: física, electrónica y software. Es el caso del subproyecto síntesis intensificada de compuestos orgánicos. En este estudio de caso, dos sustancias están acopladas por una reacción en la industria farmacéutica. Los dos reactivos $(A 1, A 2)$ se mezclan con una base de litio ( $L i$-Base) en un reactor helicoidal para producir el producto deseado $(B)$ y una sal $(L i-S a l)$ :

$$
A 1+A 2+\text { Li-Base } \rightarrow B+L i \text {-Sal }
$$

La sal precipita lo que conduce a la formación de partículas. Por desgracia, se forma una capa de incrustación sobre la superficie interna del reactor, debido a la precipitación. Después de algún tiempo, esto crece y conduce a la obstrucción del reactor por lo tanto la producción tiene que ser interrumpida para limpiar el reactor. Además, la reactividad y la temperatura de las corrientes de alimentación están sujetas a variación. La forma como CONSENS aplica el control, sensado y monitoreo ciberfisico es de la siguiente manera.

1. Aplica un sensor de incrustación en línea, que mide el espesor no invasivo-espacial y el crecimiento de la capa de incrustaciones a lo largo del reactor.

2. Sensado en línea que mide rápida y exactamente la composición de la corriente de producto (producto, residuos de reactivos, productos secundarios) con esfuerzo de calibración mínimo.

3. Utiliza algoritmos de métodos de auto adaptación

4. Utiliza herramientas para un enfoque integrado para la minería de datos y el monitoreo del desempeño.

\section{ANALISIS DE RIESGOS}

El sistema que se tiene actualmente en la empresa de estudio cumple con el estándar NOM-004STPS-1999 (Belobaba, 2015), el objetivo será agregar estándares internacionales para aumentar la seguridad. Durante el análisis del fundamento teórico se determinó un método cualitativo y cuantitativo de reducir el riesgo, este procedimiento se resumen en el diagrama de la figura 1.

Para la representación de las partes del sistema de control del módulo de seguridad, se utilizan diagramas de bloques lógicos; en la figura 2, donde ia e ib representan los medios de interconexión, eventos de iniciación (ejemplo señal de interruptor de seguridad) y eventos de salidas en los robots (ejemplo actuadores, frenos etc.); en cada proceso el sistema de mando de seguridad, tendrá que ser validado (Val).

Todo esto será monitoreado mediante un sistema llamado Nagios (Nagios, 2016), el cual es una herramienta de software libre. Este sistema monitorea servicios en equipo de cómputo, servidores en una red local o remota, ya sean equipos Windows, Linux, e incluso hardware de comunicaciones como routers y swiches. Nagios tiene como fin, facilitar la vigilancia a equipos de manera remota de la manera más fácil y óptima. En el caso de una falla, Nagios puede alertar al personal técnico del pro- 
blema, lo que permite comenzar los procesos de mantenimiento y corrección antes de que afecten a la producción final, usuarios finales o clientes, como se muestra en la figura 3. Otro punto a considerar es el diseño simple de plugins, que permiten a los usuarios desarrollar sus propias aplicaciones de monitoreo de servicios según las necesidades de cada empresa, usando herramientas como $\mathrm{C}++$, Perl, Ruby, Python, PHP, C\# etc.

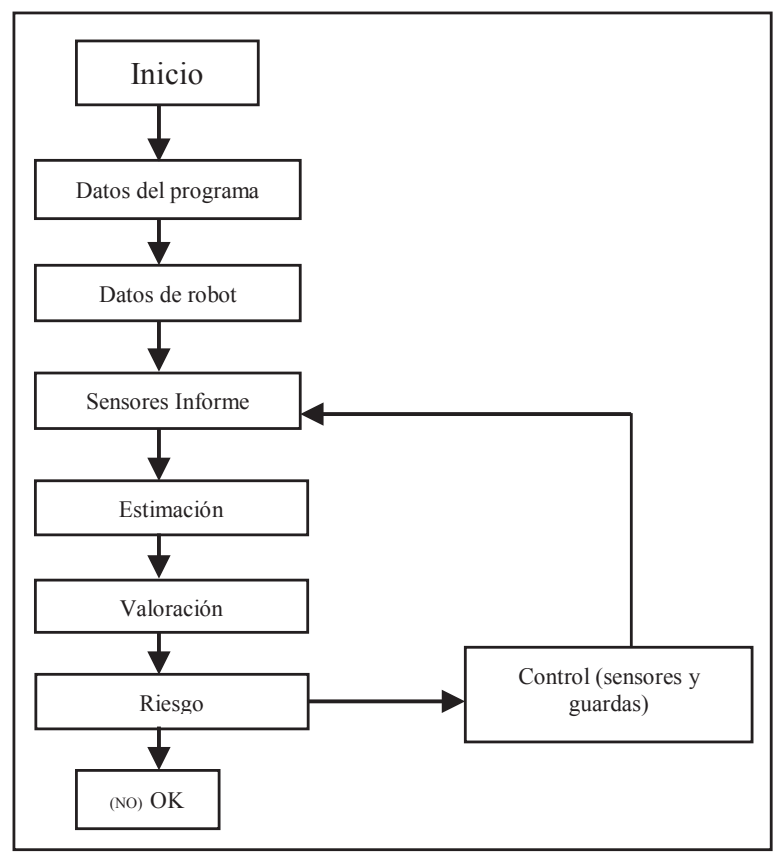

Figura 1. Proceso de análisis de riesgos al aplicar sistema ciberfisico

Fuente: elaboración propia

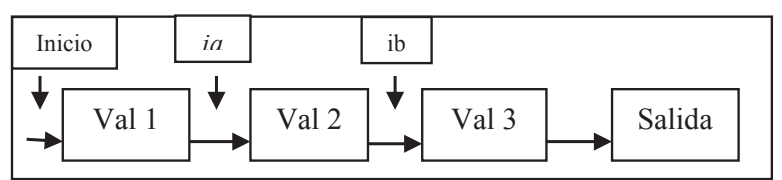

Figura 2. Módulo de seguridad

Fuente: elaboración propia

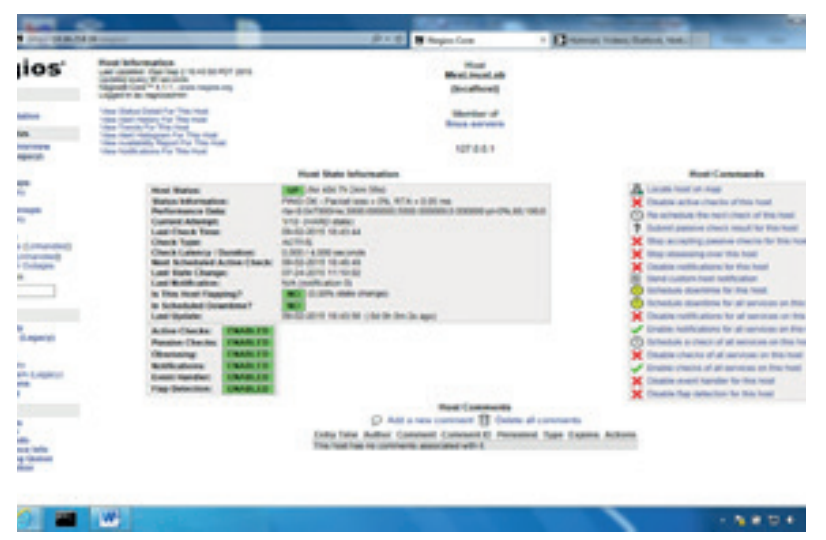

Figura 3. Sistema Nagios para monitoreo general Fuente: elaboración propia

\section{FUNDAMENTO TEÓRICO}

a. Internet de las cosas, existen simuladores de internet de las cosas (loT)( Zuehlke et al, 2010), utilizados en algunas industrias como lo es: SimpleloTSimulator: simulador de dispositivos y sensores loT el cual crea escenarios de pruebas con miles de sensores.

b. La tecnología GPS (Schwartz, 2015), permite mostrar datos importantes sobre las condiciones de trayectoria del material requerido y sus posibles rutas más óptimas. Se encuentra relacionado con el internet de las cosas, como un pegamento entre la caracterización de todo el sistema para localizar en tiempo real y notificar de forma remota material necesario en la producción. Este caso de estudio relaciona cada proceso, iniciando en el rastreo de material y terminando en el producto aeroespacial final, como lo muestra la figura 4.

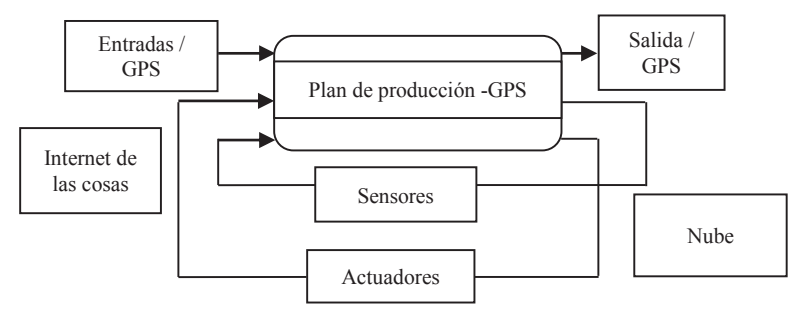

Figura 4. Interacción de GPS y el plan de producción Fuente: elaboración propia

La importancia de los sistemas de tiempo real que interaccionan con entornos físicos y estos a su vez responden dentro de un plazo de tiempo critico determinado; es decir en donde las respuestas son estrictamente respetadas, ya que una respuesta en un intervalo tardío puede tener consecuencias fatales. Una respuesta tardía significa, desde insertar un componente una milésima de distancia de donde debería ir, sería una pérdida de dinero significativa; aun peor sería el error que pusiera en peligro la vida de un operario. Por tal motivo la utilización de la tecnología GPS (Abebe, 2010), está basada en localización satelital; por la cual es posible obtener una posición global detallada representada por dos valores, latitud y longitud. La latitud es la representación en grados de la posición norte-sur con respecto al punto en la superficie en la tierra, y la longitud representa la posición este-oeste de un punto en la superficie de la tierra en grados. La importancia de GPS en los sistemas ciberfisicos radica en que toda la materia prima que se utilizara en 
la fabricación se comunicara desde el proveedor de material hasta su ensamblado y deberá comunicarse a su vez con otros dispositivos alternos, en tiempo real.

c. Red de Sensores inalámbricos (Garbarino, 2010), es un clúster de nodos sensores, los cuales miden parámetros; que pueden almacenar, procesar y retroalimentar. Los parámetros generalmente monitoreados son temperatura, presión atmosférica, movimiento, humedad, etc. La red de sensores inalámbricos tiene actualmente algunos problemas que pueden obstruir el envío de información, entre las de mayor impacto se encuentra el aumento de energía, sobrecarga de red, perdida de paquetes, etc. Generalmente la red de sensores utilizado en el internet de las cosas utiliza escalabilidad; además de utilizar redes dinámicas en las cuales, los nodos/sensores son fijos, pero también pueden ser móviles. Otro punto importante a considerar es el medio de transmisión en el cual, la comunicación entre los nodos se realiza por un medio inalámbrico

d. Sistema de tiempo real (Tobón et al, 2012), es un sistema de computación que debe reaccionar ante un evento en un tiempo preciso. En un sistema de tiempo real la característica principal es llamada plazo; que es el tiempo máximo donde la ejecución debe ser completada (Szewczyk et al, 2012). Un mensaje que llega a su destino después de su plazo o lapso de tiempo puede ocasionar problemas importantes. De tal forma que los algoritmos de tiempo real no solo deben mantener menor retraso en el envío de paquetes, también deben cumplirse dentro de un plazo de tiempo planeado.

\section{PROCESO DE PRODUCCIÓN}

Nuestra investigación se basa en el área de inserción automática (Renteria, 2011), ya que es la parte inicial del proceso de producción. Utiliza cinco procesos básicos los cuales son:

- Ey (eyelets): Inserción de un hojal donde se implantaran componentes grandes.

- Jmp (jumper): Inserción de un hilo de oro que unirá pistas o nodos, el cual puede correr en el eje $\mathrm{x} 0 \mathrm{y}$.

- Ax (axial): Inserción de componentes básicos como resistencias, capacitores, transistores etc. Este toma componentes con terminales axiales de los carretes que se alimentan en las cabezas de dispensación, y cortan las partes exteriores de los componentes de forma secuencial.

- Rx (radial): Inserción de componentes radiales como potenciómetros, diodos etc. $\mathrm{Su}$ longitud de remache es 1.2-2.2m m ajustable, con un grado de remache de 10$35^{\circ}$.

- Smt (tecnología de montaje superficial). Montaje de circuitos integrados para soldar mediante la máquina de olas.

Los robots utilizados en esta investigación son de modelo:

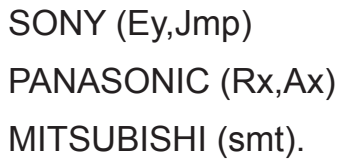

El patrón de trayectoria de la producción es como lo muestra la figura 5 .

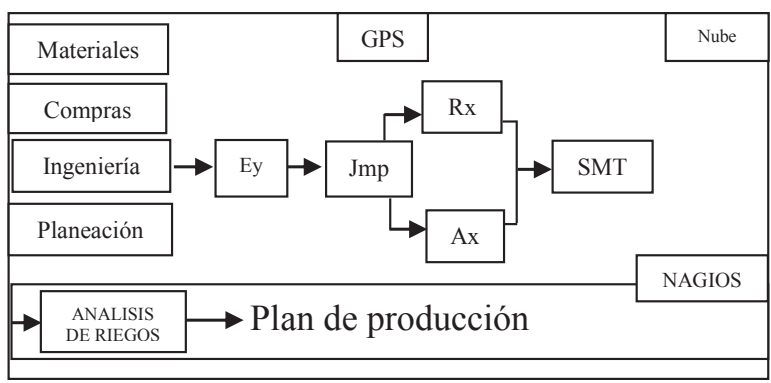

Figura 5. Trayectoria del proceso de auto inserción Fuente: elaboración propia

El lapso de tiempo en cada proceso tiene siempre un tiempo muerto (latencia), debido a los cambios de modelo, mantenimientos correctivos o preventivos del robot.

Cada proceso es dependiente del anterior y cíclico, excepto el proceso de radial y axial; ya que depende del tipo de modelo que se desea construir.

El proceso de eylets (Schraft, 2014), es un sistema donde se inserta un ojal metálico para componentes pesados, el proceso de jumper es donde se interconectan puentes de terminales o uniones de pistas electrónicas; el proceso de radial, axial se insertan componentes electrónicos como lo son resistencias, capacitores, transistores etc. Por último el proceso de SMT, es donde los componentes están soldados directamente sobre la superficie de una placa de circuito impreso. 
En la figura 6, se define el proceso de auto inserción del lote de placas de estudio. La implementación de herramientas flexibles, reconfigurables, escalables, descentralizadas y distribuidas mediante sistemas y dispositivos embebidos, hace que la producción del área de auto inserción ya no sea una producción tradicional, sino un ecosistema en donde los sistemas en red se forman por dispositivos y sistemas integrados inteligentes. La tecnología de las redes de sensores inalámbricos, computación en la nube, sistemas empotrados (Ashford, 2016), y entornos ubicuos se consideran para un buen funcionamiento en los sistemas ciberfisicos (Rajeev, 2015).

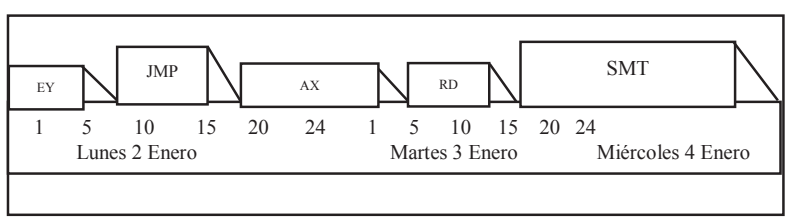

Figura 6. Tiempos de proceso, en modelo estudiado de 1000 piezas

Fuente: elaboración propia

La estructura por subsistemas, respecto a analizar cada departamento como tal, es para relacionar la interconectividad de los diferentes departamentos industriales como lo es planeación, compras, materiales, ingeniería, producción etc. De esta manera los proveedores (Ross, 2015) de componentes tendrían en tiempo real las necesidades físicas, antes de que estas sean requeridas por el departamento de compras o finanzas.
El enfoque tradicional del proceso industrial, es cuando el software se considera un todo para el sistema. Pero en el caso ciberfisico la parte de software se desarrolla sincrónicamente con la parte mecánica y electrónica.

También se utilizan brazos robóticos, en líneas de producción industrial llamados SCARA (Schraft, 2014). Estos robots SCARA son programados para realizar tareas cíclicas, en algunos procesos específicos de inserción electrónica. Se utilizan sensores para dotar de "vista", permitiendo insertar componentes muy pequeños en coordenadas específicas, en esta investigación no fueron utilizados.

\section{MODELO PROPUESTO}

El modelo propuesto se muestra en la Figura 7:

En este modelo de estudio, se utilizó un plan de producción de 1000 tableros de un modelo aeroespacial Honeywell. Se ejecutó un sistema interno de master plannig en Oracle para definir material a comprar, cantidad de salida y entrada en el departamento de almacén, cambios de ingeniería en el departamento del producto, plan de piso etc. Para el seguimiento de camiones vía GPS, se utilizó un visualizador promodel (Harrell et al, 2011) en tiempo real; esto para la localización de material inicial (componentes). También se utilizó un algoritmo de etiquetado especial de adaptación, para reconfigurar automáticamente en caso de fallos en los ro-

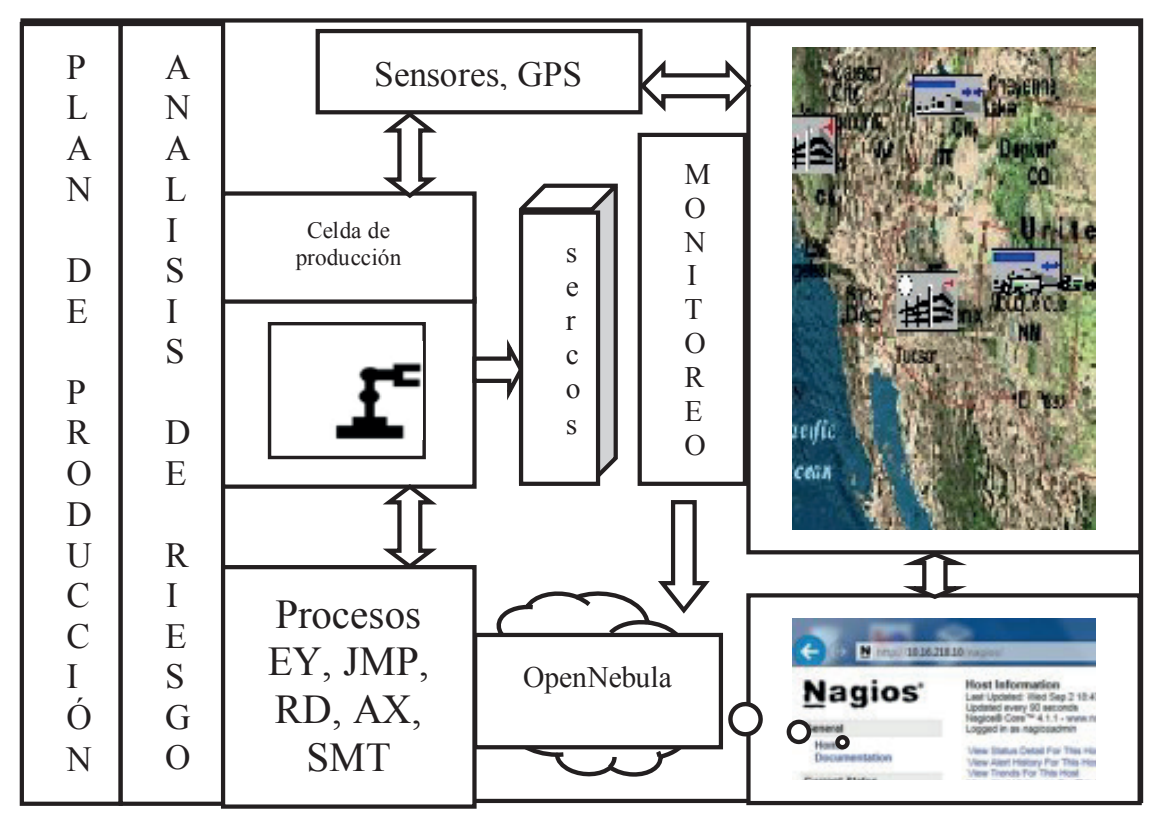

Figura 7. Modelo de Verificación de material, proceso, terminado y censado

Fuente: elaboración propia 
bots, atraso de material, corto de material etc. Este algoritmo se define en un dominio finito $D$ y una función $f: D \rightarrow$ donde la función $f$ encuentra el mejor valor de la función $f$ en el dominio $D$. De tal forma que la posición a insertar $(x)$ es: $x \in D$ donde $f(x) \leq$ $\mathrm{f}(\mathrm{y}) \forall \mathrm{y} \in \mathrm{D}$. Donde $\mathrm{D}$ es el total del lote a producir $\mathrm{y}$ $E$ es el componente a insertar.

Para definir el número de fallos, se debe mediante:

$$
\operatorname{Max}\{f(x)\}=-\operatorname{Min}\{-f(x)\}
$$

La forma para retroalimentar el algoritmo es mediante la función $f$ donde $x^{\wedge} \in D$ para $\exists E(x)$, tal que sí $\in E(x), \Rightarrow f(x) \leq f(y)$ donde la función $f(x)=g(e(x))$ que se denomina función objetivo. La cual define $y$ compara los fallos ocurrentes con el plan de producción inicial y este lo reprograma en todos los departamentos incluyendo mantenimiento correctivo y preventivo de los robots. Este mismo algoritmo se utilizó al momento de programar las máquinas de auto inserción ya que el crear cabezas dispensadoras y asignarle los componentes a cada proceso era una tarea manual, en el cual el operador tenía que verificar una lista de los componentes a insertar y ponerlos manualmente en el carrete de llenado de las maquinas; dando como consecuencias errores de componentes, posibles accidentes en los dedos generalmente (Estadísticas Gubernamentales, 2016), además de retardos de producción que se traducen a dinero. Los componentes del modelo son:

Módulo nube: La comunicación de materiales, maquinaria, operarios debe estar bajo la plataforma de una nube, donde se almacenara información, virtualizará (debido a infraestructuras distribuidas), monitoreo y se le dará seguimiento de seguridad a todo el sistema ciberfisico. Es posible que existan nubes remotas, comunicándose con la nube principal; y comparta recursos y se reconfigure dinámicamente según la demanda. Para esto se utilizó OpenNebuba (Toraldo, 2013); es de código abierto y permite la gestión de infraestructuras en sistemas distribuidos y heterogéneos; incluyendo clúster, nubes hibridas etc. También tiene la capacidad de Grid Computacional (Yang Liu et al, 2017) es decir, manejar redes de clúster para información. Es por todo esto, que es necesario una nube dedicada; con estructura y configuraciones especificas la cual pueda reconfigurarse automáticamente. En este caso de estudio no fue posible probar dicha nube, ya que la empresa por cuestiones de seguridad no permite información al exterior, solo se hicieron pruebas simuladas.

\section{Módulo traductor de protocolos}

Es un área de investigación abierta para analizar y sincronizar todos los protocolos en los sistemas ciberfisicos, estos protocolos en la industria son utilizados debido a la gran complejidad de datos, comunicación y conectividad, en este caso de estudio se utilizó:

a. SERCOS (Trosky, 2010): El bus está optimizado para el control de movimiento determinístico de alta velocidad, que se requiere para la sincronización exacta de varias unidades. Básicamente SERCOS, es una interface abierta de tiempo real; estandarizada para la comunicación de controles automatizados (servomecanismos, controles neumáticos e hidráulicos, sistemas de visión, codificadores y dispositivos periféricos distribuidos. Se utilizó una versión SERCOS II utilizada en Honeywell Aerospace and Defense de Guangzhou China (ZоБарановс et al, 2016).

\section{b. Protocolo en Tiempo Real, RTP}

El objetivo de RTP es transmitir sobre IP de datos que estén sujetos a las limitaciones de tiempo real (audio, video). Básicamente implementa las secuencias de paquetes IP para reorganizar la información de voz o de video.

Módulo de Voz: Es el componente donde se le dan instrucciones de emergencia o de actividad a toda la maquinaria robotizada incluyendo el sistema de envió de status de producción vía celular, utilizando Bitvoicer. Bitvoicer es un software que permite el reconocimiento de voz (Groover, 2014), para así permitir utilizar micro controladores; dicho software permite el control de los procesamientos y el análisis de voz. Básicamente se selecciona el tipo de dato que será el comando que se envié al controlador, dentro del cuadro de la secuencia, como lo muestra la figura 8.

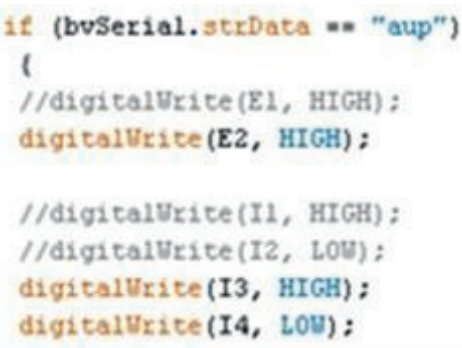

Figura 8. Ejemplo que indica el movimiento que realizara el brazo mediante voz

Fuente: elaboración propia 
Módulo Galileo: Se refiere a la interfaz que traduce los movimientos registrados en la cámara, sistema móvil y voz, para mover los servomotores del brazo robótico. Este a su vez está conectado a una placa Raspberry pi (Richardson, 2016), que dará salida vía wifi a la información requerida ya sea a un celular, a Nagios o la nube. Galileo Arduino (Claus, 2011), se programa mediante el lenguaje de programación Arduino (basado en Wiring) y el entorno de desarrollo Arduino (basado en Procesing (Shiffman, 2011)). Arduino, es una plataforma de hardware libre basada en un micro controlador diseñada para facilitar el uso de la electrónica en general, consta de puertos de entrada y salida como lo muestra la siguiente codificación en la figura 9.

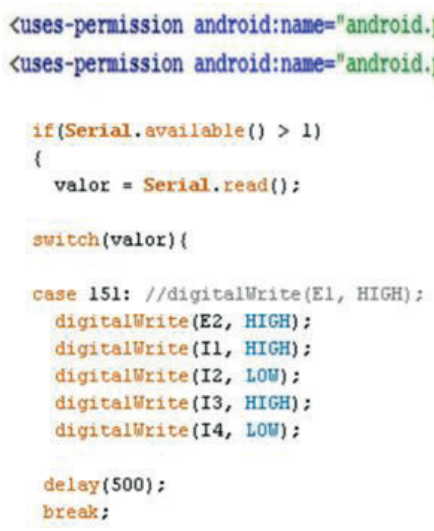

Figura 9. Ejemplo que indica el movimiento que realizara el brazo mediante voz

Fuente: elaboración propia

Con estos módulos, se da la integración del monitoreo y los pronósticos de fallos del sistema, desde cortos de material, retraso de la materia prima, fallos de robot especificando tipo de fallo y estimación, cambios de ingeniería etc. Estos son los parámetros que cambian automáticamente el plan de producción y reprograman el análisis de riesgo que ayudan a detectar y aislar fallos en el sistema.

De tal forma que la formulación de nuestro modelo está definido por $x(k+1)$ donde la posición a insertar $(x)$ está dada por una variable discreta en el tiempo $(k)$ la cual tiene parámetros desconocidos que pueden provocar un error: por lo tanto $x(k+1)=$ $f(k, x(k), y(k), e(k))$, tal que $y$ es la salida esperada $y$ e el componente a insertar.

Dado que el sistema incluye área de producción (específicamente auto inserción), rastreo de componentes, situación de robots, estimación de plan de producción, monitoreo general del sistema, historial en la nube etc. Los pronósticos respecto a los estados, parámetros, entradas del sistema son llamados eventos $\boldsymbol{R}$ donde $n$ define el instante de tiempo donde el robot inserta el componente $R^{n 1} \times R^{n 2} \times R^{n}: T$ y la función objetivo $\mathrm{g}(\mathrm{e}(\mathrm{x}))=$ $P P=\Delta(0,1)$ la función objetivo es el resultado de acción del robot evaluada en verdadero o falso. La toma de decisiones en la mayoría de las empresas electrónicas, solo utilizan un plan estratégico donde no relacionan directamente algo físico; aunque su resultado de plan, se lleva a cabo simultáneamente pero independiente de su sistema de análisis.

\section{CONCLUSIONES}

El integrar diferentes procesos de producción y sincronízalos en un solo algoritmo ciberfisico es complejo, ya que intervienen variables que se entrelazan y repercuten en distintas áreas del proceso. La métrica para verificar si funciona dicho algoritmo ciberfisico; fue tomando tiempos de los diferentes procesos (ey,ax,rd,smt) en un lote de producción de 1000 piezas del tablero "Flow" que es parte de un instrumento de velocidad vertical llamado variómetro. Los tiempos se redujeron de 390 horas (figura 10), a un promedio de 280 horas (figura 11). Aun se requiere hacer más pruebas para verificar el comportamiento y analizar en diferentes lotes de producción es decir en diferentes tableros aeronáuticos.

En este documento se analizó la combinación de software y hardware relacionado a un sistema ciberfisico, utilizado en una empresa aeroespacial; específicamente en el área de auto inserción.

Debido a que este caso de estudio es respecto a la adaptabilidad que debe tener el sistema, incluyendo robots, material, operadores y los diferentes departamentos de manufactura debe ser analizado en diferentes fases. Ya que la producción requiere constantemente de cambios rápidos. Esta configuración ciberfisica contiene paramentos que pueden ser aplicados en el trasporte de material, programación de máquinas etc. Se necesitan hacer más pruebas y análisis, para determinar el ahorro de tiempo, energía, dinero y seguridad que los sistemas ciberfisicos pueden aportar al campo industrial.

Es necesario hacer también un análisis de patrones utilizando sistemas con BigData (Schönberger, 2015). En este artículo se utiliza la arquitectura orientada a servicios, la explotación del internet de las Cosas, que han impulsado la tecnología ciberfisica (Rajeev, 2015). Los nuevos retos que implican las nuevas tecnologías y la comunicación con la tecnología y protocolos anteriores, es todo un reto. 


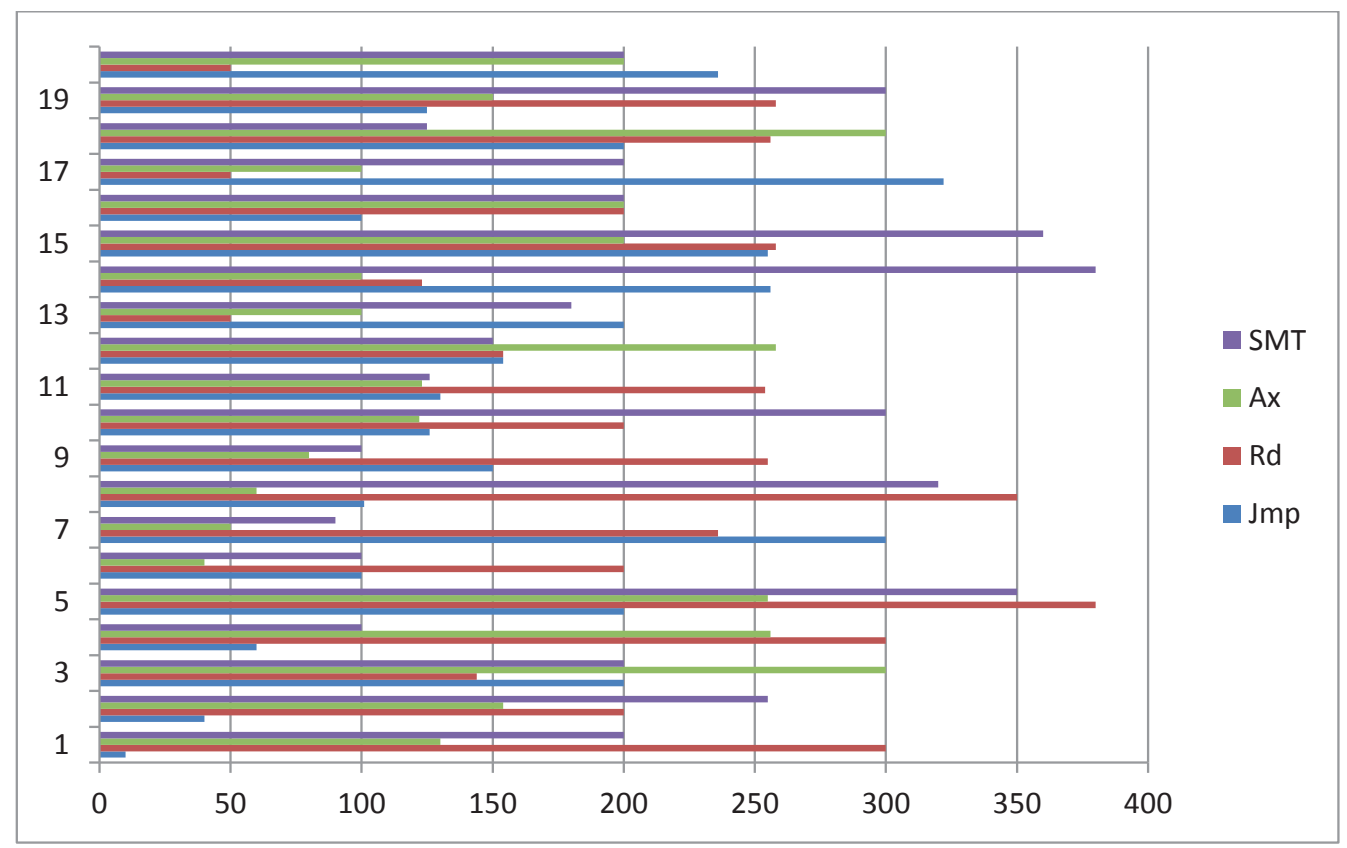

Figura 10. Resultados de tiempos en los diferentes procesos de producción, antes de aplicar el sistema ciberfisico propuesto

Fuente: elaboración propia

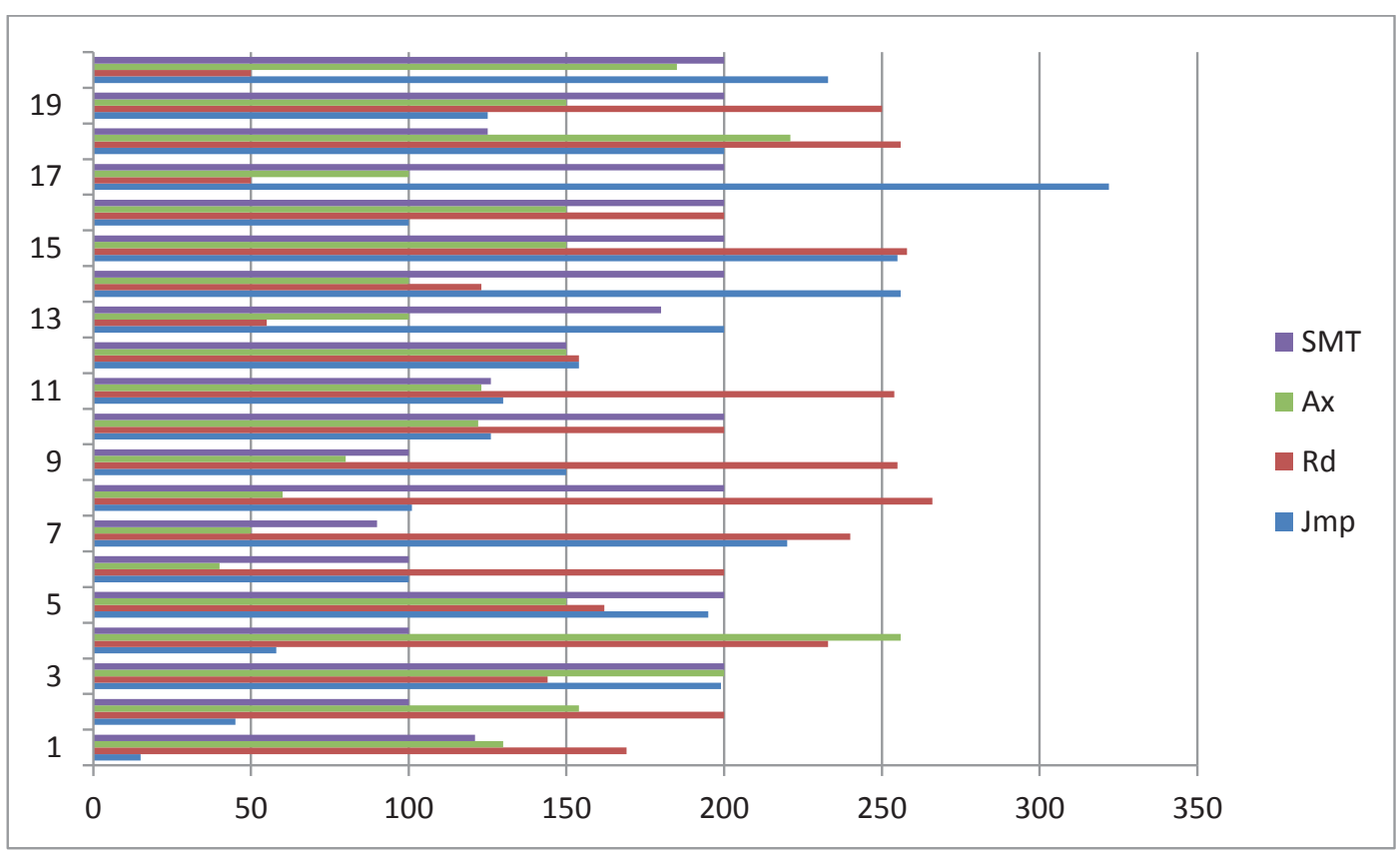

Figura 11. Resultados de tiempos en los diferentes procesos después de aplicar el sistema ciberfisico propuesto Fuente: elaboración propia

El rápido avance en la automatización industrial (Roca, 2014), los sistemas inteligentes, los sistemas empotrados y ahora con el nuevo paradigma de los sistemas ciberfisicos es necesario hacer más estudios e investigación ya que la tradicional forma de producir y manufacturar deberá adaptarse a dichos avances.
Existe como trabajo futuro el uso de Óculos Rift ( Parisi, 2016), el cual es una herramienta base, en la complejidad de los sistemas Ciber Físicos; ya que consiste en integrar y aplicar controles, comunicaciones, sensores, verificación y seguridad a un conjunto de sistemas interactuando entre sí. A pesar que esto es visto como futuro, actualmente ya las 
compañías telefónicas están dando a sus clientes el uso de estos lentes, lo cual nos permitirá a un futuro muy corto poder utilizar esta tecnológica ya que podremos tener un sistema embebidos, que generalmente utilizan procesadores muy básicos con memorias pequeñas para minimizar los costos. El sistema Óculos Rift estaría dentro de un sistema embebido, real y ciberfisico al mismo tiempo por esa razón es nuestro interés analizarlo en el futuro. Analizar los sistemas ciberfisicos es complejo ya que es capaz de llevar acabo procesos en tiempo real en conjunto con diferentes protocolos, sensores y sistemas. Sin embargo el resultado que se busca es anular los procedimientos que resulten peligrosos para un operador, errores técnicos mecánicos, pérdidas de tiempo y material etc.

\section{REFERENCIAS BIBLIOGRÁFICAS}

[1] Abebe E. (2010). GPS and GSM based real time vehicle locater component framework: For GIS enabled fleet management systems. Germany: VDM Verlag.

[2] Ashford L. (2016). Introduction to Embedded Systems - A Cyber-Physical Systems Approach. Berkely, CA, USA: MIT Press.

[3] Renteria A. (2011). Robótica Industrial Fundamentos y aplicacaciones. España: Mc Graw Hill.

[4] Belobaba Pouth (2015). The Global Airline Industry. Massachussets, USA: Wiley.

[5] Beth C. (2011). CMMI for Development: Guidelines for Process Integration and Product Improvement (SEI Series in Software Engineering). USA: Addison-Wesley.

[6] Claus K (2011). Arduino: Hard and Software Open Source Plattform. USA: KHnel

[7] Federación mexicana de la industria aeroespacial. (1 Abril 2016) Recuperado de : http://www.femia.com.mx/

[8] Estadisticas Gubernamentales; accidentes y enfermedades de trabajo. (4 Marzo 2016). Recuperdado de: http://www.stps.gob.mx/bp/ secciones/dgsst/estadisticas.htm

[9] Garbarino J. (2010). Protocolos para Redes Inalámbricas de Sensores: Conciencia de energía y técnicas de diseminación en protocolos de red para redes inalámbricas de sensores. España: Editorial Académica Española.
[10] Greengard S. (2015). The Internet of Things. Cambridge, Ma, USA: The MIT Press.

[11] Groover F.(2014). Automation, Production Systems, and Computer-Integrated Manufacturing. USA: Prentice Hall.

[12] Harrell C. \& Gosh Biman (2011). Simulation Using ProModel. USA: Mc Graw Hill.

[13] INEGI. Indicadores Mensuales de la Actividad Industrial en México. (6 febrero 2016). Recuperdado de: http://www.inegi.org.mx/

[14] Kowther F. (4 Enero 2016). European Union's Horizon 2020 research and innovation programme under grant agreement $N^{\circ} 636942$. Germany: Springer.

[15] Kuhrmann M. \& Das T. (2016). V-Mode//® XT (Informatik im Fokus). Germany: Springer.

[16] Nagios. Oficial. (1 septiembre 2016). Recuperado de https://www.nagios.org/

[17] Parisi. (2016). Learning Virtual Reality: Developing Immersive Experiences and Applications for Desktop, Web, and MobilePaperback. Sebastopol, CA., USA: O'Reilly Media.

[18] Rajeev A. (2015). Principles of Cyber-Physical Systems. Cambridge, Massachussets, USA: MIT Press.

[19] Richardson M. (2016). Getting Started With Raspberry Pi. San Francisco, CA., USA: Maker Media.

[20] Roca A. (2014). Control automático de procesos industriales. México: Editorial Díaz de Santos.

[21] Ross J. (2015). Methods for shipping freight US6332098 B2. USA: Prentice Hall.

[22] Sang A. (Eds). (2015). Applied Cyber-Physical Systems. New York, USA: Springer.

[23] Szewczyk R. \& Culler D. (2012). Supporting aggregate queries over ad-hoc wireless sensor networks. In Mobile Computing Systems and Applications. USA: MIT Press.

[24] Schwartz M. (2015). GSM \& GPS Projects With Arduino. USA: Maker Media.

[25] Schraft D. (2014). Pcb Assembly Systems, 1989: The International Directory of Smt/ Insertion Equipment. USA: Springer-Verlag.

[26] Schönberger F. (2015). Big Data: A Revolution That Will Transform How We Live, Work, and Think.. USA: Eamon. 
[27] Shiffman C. (2011). Learning Processing: A Beginner's Guide to Programming Images, Animation, and Interaction. USA: Morgan.

[28] Tobón V. \& Gaviria G. (2012). Análisis de métricas de calidad de servicio para la configuración del protocolo csma/ca en redes de sensores inalámbricas de área corporal. USA: Prentice.

[29] Trosky V. (2010). SERCOS III. IEC TC/SC 65C. EC/PAS 62410 Ed. 1.0 :2005, Real-time Ethernet. USA: Distributed through American National Standards Institute (ANSI).
[30] Toraldo G. (2013). OpenNebula 3 Cloud Computing. USA: Springer.

[31] Yang Liu \& Yu Peng (2017). Review on Cyberphysical Systems. IEEE/CAA JOURNAL OF AUTOMATICA SINICA, 4 (1), 27-40. DOI: 10.1109/JAS.2017.7510349

[32] Zuehlke, D. \& Sorgheit A. (2010). Towards a Factory-of-Things. USA: Princeton.

[33] ZоБарановс\&Краснопрошин(2016). ОЦЕНКА ЭФФЕКТИВНОСТИ ПОДРАЗДЕЛЕНИЙ РАСПРЕДЕЛЕННЫХ КОМПАНИЯХ DOІ: 10.1144/JES.2016.1067287. Google Scholar. 